\title{
Fourth Hankel Determinant for a Subclass of Starlike Functions Based on Modified Sigmoid
}

\author{
Wali Khan Mashwani $\left(\mathbb{D},{ }^{1}\right.$ Bakhtiar Ahmad $\left(\mathbb{D},{ }^{2}\right.$ Nazar Khan, ${ }^{3}$ Muhammad Ghaffar Khan $(\mathbb{D})$, \\ Sama Arjika $\mathbb{D}^{4,5}$ Bilal Khan $\mathbb{D}^{4},{ }^{6}$ and Ronnason Chinram $\mathbb{D}^{7}$ \\ ${ }^{1}$ Institute of Numerical Sciences, Kohat University of Science and Technology, Kohat, Pakistan \\ ${ }^{2}$ Govt. Degree College Mardan, 23200 Mardan, Pakistan \\ ${ }^{3}$ Department of Mathematics, Abbottabad University of Science and Technology, Abbottabad 22010, Pakistan \\ ${ }^{4}$ Department of Mathematics and Informatics, University of Agadez, Agadez, Niger \\ ${ }^{5}$ International Chair of Mathematical Physics and Applications (ICMPA-UNESCO Chair), University of Abomey-Calavi, \\ Post Box 072, Cotonou 50, Benin \\ ${ }^{6}$ School of Mathematical Sciences and Shanghai Key Laboratory of PMMP, East China Normal University, 500 Dongchuan Road, \\ Shanghai 200241, China \\ ${ }^{7}$ Algebra and Applications Research Unit, Division of Computational Science, Faculty of Science, Prince of Songkla University, \\ Hat Yai, Songkhla 90110, Thailand
}

Correspondence should be addressed to Sama Arjika; rjksama2008@gmail.com

Received 22 May 2021; Revised 11 August 2021; Accepted 30 November 2021; Published 16 December 2021

Academic Editor: John R. Akeroyd

Copyright ( 2021 Wali Khan Mashwani et al. This is an open access article distributed under the Creative Commons Attribution License, which permits unrestricted use, distribution, and reproduction in any medium, provided the original work is properly cited.

In our present investigation, we obtain the improved third-order Hankel determinant for a class of starlike functions connected with modified sigmoid functions. Further, we investigate the fourth-order Hankel determinant, Zalcman conjecture, and also evaluate the fourth-order Hankel determinants for 2-fold, 3-fold, and 4-fold symmetric starlike functions.

\section{Introduction and Motivation}

Denoted by $\mathscr{A}$, the class of functions $f$ which are analytic in

$$
\mathbb{U}=\{z: z \in \mathbb{C} \text { and }|z|<1\} \text {, }
$$

and are of the form

$$
f(z)=z+\sum_{n=2}^{\infty} a_{n} z^{n}(\forall z \in \mathbb{U}) .
$$

Also, let $\mathcal{S}$ be a subclass of class $\mathscr{A}$, containing all univalent functions in $\mathbb{U}$, and be normalized by the conditions

$$
\begin{aligned}
& f(0)=0, \\
& f^{\prime}(0)=1 .
\end{aligned}
$$

In 1916, working on the coefficients $a_{n}$ of class $\delta$, Bieberbach conjectured that

$$
\left|a_{n}\right| \leq n(n=2,3, \cdots,)
$$

which was proved by De Branges in 1984 (see [1]). From 1916 to 1984 , for some subclasses of $\mathcal{S}$, many researchers used different techniques and established a number of results. The classes $\mathcal{S}^{*}, \mathscr{K}$, and $\mathscr{R}$, namely, the classes of convex, starlike, and bounded turning functions, respectively, are some major subclasses of the class $\mathcal{S}$. These classes are defined as follows: 


$$
\begin{gathered}
\mathcal{S}^{*}=\left\{f \in \mathcal{S}: \mathfrak{R e}\left(\frac{z f^{\prime}(z)}{f(z)}\right)>0(\forall z \in \mathbb{U})\right\}, \\
\mathscr{K}=\left\{f \in \mathcal{S}: \mathfrak{R e}\left(\frac{\left(z f^{\prime}(z)\right)^{\prime}}{f^{\prime}(z)}\right)>0(\forall z \in \mathbb{U})\right\}, \\
\mathscr{R}=\left\{f \in \mathcal{S}: \mathfrak{R e}\left[f^{\prime}(z)\right]>0(\forall z \in \mathbb{U})\right\} .
\end{gathered}
$$

Furthermore, we say that an analytic function $f_{1}(z)$ is subordinated to $f_{2}(z)$ in $\mathbb{U}$ and is symbolically written as

$$
f_{1}(z) \prec f_{2}(z)(\forall z \in \mathbb{U}),
$$

if there exists a Schwartz function $u(z)$ with properties that

$$
\begin{gathered}
|u(z)| \leq 1, \\
u(0)=1,
\end{gathered}
$$

such that

$$
f_{1}(z)=f_{2}(u(z))
$$

Moreover, if $f_{2}(z)$ is in the class $\mathcal{S}$. Due to $[2,3]$, we get the following equivalence relation

$$
\begin{aligned}
& f_{1}(0)=f_{2}(0), \\
& f_{1}(\mathbb{U}) \subseteq f_{2}(\mathbb{U}) .
\end{aligned}
$$

Now, by using the principle of subordination, a generalized set of the classes $\mathcal{S}^{*}, \mathscr{K}$, and $\mathscr{R}$ are given, respectively, as follows:

$$
\begin{aligned}
& \mathcal{S}^{*}(\psi)=\left\{f \in \mathcal{S}: \frac{z f^{\prime}(z)}{f(z)} \prec \psi(z)=\frac{1+z}{1-z}(\forall z \in \mathbb{U})\right\}, \\
& \mathscr{K}(\psi)=\left\{f \in \mathcal{S}: \frac{\left(z f^{\prime}(z)\right)^{\prime}}{f^{\prime}(z)} \prec \psi(z)=\frac{1+z}{1-z}(\forall z \in \mathbb{U})\right\}, \\
& \mathscr{R}(\psi)=\left\{f \in \mathcal{S}: f^{\prime}(z)<\psi(z)=\frac{1+z}{1-z}(\forall z \in \mathbb{U})\right\} .
\end{aligned}
$$

By changing the right-hand side in (10), several familiar classes can be obtained such as if we keep $\psi(z)=(1+A z) /$ $(1+B z)$, we get the class of starlike functions associated with the Janowski functions (see [4]). Moreover, if we take

$$
\begin{gathered}
A=1, \\
B=1-2 \alpha(0<\alpha<1),
\end{gathered}
$$

then, we have a class of starlike functions of order $\alpha$ (see [5]). Also, for the choice of $\psi(z)=1+\left(2 / \pi^{2}\right)$ $(\log ((1+\sqrt{z}) /(1-\sqrt{z})))^{2}$, a corresponding class of starlike functions is obtained, which was introduced by Ronning (see [6]). Furthermore, if we take $\psi(z)=\sqrt{1+z}$, the class of starlike functions related with the lemniscate of Bernoulli domain is resulted which was introduced and investigated by Jangteng et al. [7, 8]. Next, if we take $\psi(z)=1+\sin (z)$, the family of starlike functions connected with the sine function is obtained (see [9]). Mendiratta et al. [10] obtained a subclass of strongly starlike functions associated with exponential functions for the choice of $\psi(z)=e^{z}$. Sharma et al. [11] derived a class of starlike functions associated with a cardioid domain by taking $\psi(z)=1+(4 / 3) z+(2 / 3) z^{2}$.

Moreover, several more subclasses of starlike functions have recently been presented in [12-15] through selecting specific functions for $\psi$, like functions associated with conic domains, shell-like curves associated with Fibonacci numbers, and functions related with Bell numbers.

Lately, based on the techniques of Ma and Minda [16], Goel and Kumar in [17] defined the class $\mathcal{S}_{S G}^{*}$, based on the subordination principle, as follows:

$$
\frac{z f^{\prime}(z)}{f(z)} \prec \frac{2}{1+e^{-z}}(\forall z \in \mathbb{U})
$$

and studied its various important geometric properties.

For a function $f$ of the form (2), Pommerenke $[18,19]$ defined Hankel determinant $H_{q, n}(f)$, parameter $q$, with $n$ $\in \mathbb{N}=\{1,2,3, \cdots\}$, as follows:

$$
H_{q, n}(f)=\left|\begin{array}{cccc}
a_{n} & a_{n+1} & \cdots & a_{n+q-1} \\
a_{n+1} & a_{n+2} & \cdots & a_{n+q} \\
\vdots & \vdots & \cdots & \vdots \\
a_{n+q-1} & a_{n+q} & \cdots & a_{n+2 q-2}
\end{array}\right| .
$$

The growth of $H_{q, n}(f)$ for fixed integer $q$ and $n$ was evaluated for different subfamilies of univalent functions. Jangteng et al. $[7,20]$ investigated the sharp bound of the determinant $H_{2,2}(f)=\left|a_{2} a_{4}-a_{3}^{2}\right|$ for each of the classes $\mathscr{K}$, $\mathcal{S}^{*}$, and $\mathscr{R}$, while sharp estimation for the family of closeto-convex functions is still unknown (see [21]). On the other hand, Krishna et al. [22] proved the best estimate of $\left|H_{2,2}(f)\right|$ for the class of Bazilevic functions. More detailed work on $H_{2,2}(f)$ can be seen in [23-27] and also the references cited therein.

The determinant,

$$
H_{3,1}(f)=\left|\begin{array}{lll}
1 & a_{2} & a_{3} \\
a_{2} & a_{3} & a_{4} \\
a_{3} & a_{4} & a_{5}
\end{array}\right|,
$$

is known as third-order Hankel determinant and the estimation of this determinant $\left|H_{3,1}(f)\right|$ is more difficult and hence a potential attraction for a lot of researchers who focus on this field. In 1966-1967, Pommerenke defined this Hankel determinant but was an open problem till 2010. In 2010, 
Babalola [28] was the first researcher who worked on $H_{3,1}($ $f$ ) and successfully obtained the upper bound of $\left|H_{3,1}(f)\right|$ for the functions belonging to the classes $\delta^{*}, \mathscr{K}$, and $\mathscr{R}$. A few mathematicians further expanded on this work to include other subclasses of holomorphic and univalent functions (see for example [29-35]). Zaprawa [36] enhanced their work in 2017 by demonstrating

$$
\left|H_{3,1}(f)\right| \leq \begin{cases}1, & \left(f \in \mathcal{S}^{*}\right), \\ \frac{49}{540}, & (f \in \mathscr{K}), \\ \frac{41}{60}, & (f \in \mathscr{R}),\end{cases}
$$

and asserted that these inequalities are still nonsharp. Additionally, for the sharpness, he thought about the subfamilies of $\mathcal{S}^{*}, \mathscr{K}$, and $\mathscr{R}$ comprising of functions with $m$-fold symmetry and acquired the sharp bounds. Recently, in 2018, Kowalczyk et al. [37] and Lecko et al. [38] got the sharp inequalities which are

$$
\begin{gathered}
\left|H_{3,1}(f)\right| \leq \frac{4}{135}, \\
\left|H_{3,1}(f)\right| \leq \frac{1}{9},
\end{gathered}
$$

for the classes $\mathscr{K}$ and $\mathcal{S}^{*}(1 / 2)$, respectively, where the symbol $\mathcal{S}^{*}(1 / 2)$ indicates the family of starlike functions of order $1 / 2$.

The main goal of this paper is to investigate the necessary and sufficient conditions for functions to get into the class $\delta_{S G}^{*}$ in the form of coefficient inequality, convolution results, and the essential third-order Hankel determinant for this class in (6) and also for its 2-, 3-, and 4-fold symmetric functions.

\section{A Set of Lemmas}

Let $\mathscr{P}$ be the family of functions $p$ that are holomorphic in $\mathbb{U}$ with $\operatorname{Re}(p(z))>0$ and its series form is as follow:

$$
p(z)=1+\sum_{n=1}^{\infty} c_{n} z^{n}(\forall z \in \mathbb{U}) .
$$

Lemma 1. If $p \in \mathscr{P}$ and it is of the form ((19)), then,

$$
\begin{gathered}
\left|c_{n}\right| \leq 2(n \geq 1), \\
\left|c_{n+k}-\delta c_{n} c_{k}\right| \leq 2(0 \leq \delta \leq 1), \\
\left|c_{2}-\xi c_{1}^{2}\right| \leq 2 \max \{1 ;|2 \xi-1|\}(\xi \in \mathbb{C}) .
\end{gathered}
$$

Further results related to Lemma 1 can be found in $[39,40]$.
Lemma 2 (see [41]). Let $p \in P$ have the series expansion of the form ((19)). Then, for $x, \sigma, \rho \in \bar{\cup}=\mathbb{U} \cup\{1\}$,

$$
\begin{gathered}
2 c_{2}=c_{1}^{2}+x\left(4-c_{1}^{2}\right), \\
4 c_{3}=c_{1}^{3}+2\left(4-c_{1}^{2}\right) c_{1} x-c_{1}\left(4-c_{1}^{2}\right) x^{2}+2\left(4-c_{1}^{2}\right)\left(1-|x|^{2}\right) \sigma, \\
8 c_{4}=c_{1}^{4}+\left(4-c_{1}^{2}\right) x\left[c_{1}^{2}\left(x^{2}-3 x+3\right)+4 x\right]-4\left(4-c_{1}^{2}\right) \\
\cdot\left(1-|x|^{2}\right)\left[c(x-1) \sigma+\bar{x} \sigma^{2}-\left(1-|\sigma|^{2}\right) \rho\right] .
\end{gathered}
$$

Lemma 3 (see [42]). Let $m, n, l$ satisfy the inequalities $0<m$ $<1,0<r<1$, and

$$
\begin{aligned}
& 8 r(1-r)\left[(m n-2 l)^{2}+(m(r+m)-n)^{2}\right] \\
& +m(1-m)(n-2 r m)^{2} \leq 4 m^{2}(1-m)^{2} r(1-r) .
\end{aligned}
$$

If $p \in \mathscr{P}$ and has power series (19), then,

$$
\left|l p_{1}^{4}+r p_{2}^{2}+2 m p_{1} p_{3}-\frac{3}{2} n p_{1}^{2} p_{2}-p_{4}\right| \leq 2 .
$$

\section{Improve Upper Bound $H_{3,1}(f)$ for the Class $\mathcal{S}_{S G}^{*}$}

To prove Theorem 6, we need the following two lemmas (Lemma 4 and Lemma 5).

Lemma 4 (see [43]). If $f \in \mathcal{S}_{S G}^{*}$ and is of the form (2), then,

$$
\left|a_{3}-a_{2}^{2}\right| \leq \frac{1}{4} .
$$

Lemma 5 (see [43]). Let $f \in \mathcal{S}_{S G}^{*}$ be of the form (2), and then,

$$
\left|a_{2} a_{3}-a_{4}\right| \leq \frac{1}{6} .
$$

We now state and prove Theorem 6 .

Theorem 6. Let $f \in \mathcal{S}_{S G}^{*}$ be of the form (2), and then,

$$
\begin{gathered}
\left|a_{6}\right| \leq \frac{21797}{57600} \simeq 0.37842, \\
\left|a_{7}\right| \leq \frac{1424429}{2073600} \simeq 0.68694, \\
\left|a_{2} a_{4}-a_{3}^{2}\right| \leq \frac{1}{16} .
\end{gathered}
$$

The result is sharp for function

$$
f(z)=z \exp \left(\int_{0}^{z} \frac{e^{t^{2}}-1}{t\left(e^{t^{2}}+1\right)} d t\right)=z+\frac{1}{4} z^{3}+\cdots
$$

Proof. Since $f \in \mathcal{S}_{S G}^{*}$, then, there exists a Schwarz function $w(z)$, given in (7) such that 


$$
\frac{z f^{\prime}(z)}{f(z)}=\frac{2}{1+e^{-w(z)}} .
$$

Let

$$
\begin{gathered}
\Psi(w(z))=\frac{2}{1+e^{-w(z)}}, \\
k(z)=1+c_{1} z+c_{2} z^{2}+\cdots=\frac{1+w(z)}{1-w(z)} .
\end{gathered}
$$

Obviously, the function $k(z) \in \mathscr{P}$ and

$$
w(z)=\frac{k(z)-1}{k(z)+1}
$$

This gives

$$
w(z)=\frac{k(z)-1}{k(z)+1}=\frac{c_{1} z+c_{2} z^{2}+c_{3} z^{3}+\cdots}{2+c_{1} z+c_{2} z^{2}+c_{3} z^{3}+\cdots} .
$$

From (31) and (34), we have

$$
\begin{aligned}
\frac{2}{1+e^{-w(z)}}= & 1+\frac{1}{4} c_{1} z+\left(\frac{1}{4} c_{2}-\frac{1}{8} c_{1}^{2}\right) z^{2}+\left(\frac{11}{192} c_{1}^{3}-\frac{1}{4} c_{2} c_{1}+\frac{1}{4} c_{3}\right) z^{3} \\
& +\left(-\frac{3}{128} c_{1}^{4}+\frac{11}{64} c_{1}^{2} c_{2}-\frac{1}{4} c_{3} c_{1}-\frac{1}{8} c_{2}^{2}+\frac{1}{4} c_{4}\right) z^{4}+\cdots
\end{aligned}
$$

while

$$
\begin{aligned}
\frac{z f^{\prime}(z)}{f(z)}= & 1+a_{2} z+\left(2 a_{3}-a_{2}^{2}\right) z^{2}+\left(a_{2}^{3}-3 a_{2} a_{3}+3 a_{4}\right) z^{3} \\
& +\left(-a_{2}^{4}+4 a_{2}^{2} a_{3}-4 a_{2} a_{4}-2 a_{3}^{2}+4 a_{5}\right) z^{4} \\
& +\left(5 a_{6}-5 a_{2} a_{5}+a_{2}^{5}-5 a_{3} a_{4}-5 a_{2}^{3} a_{3}+5 a_{2}^{2} a_{4}+5 a_{2} a_{3}^{2}\right) z^{5} \\
& +\left(\begin{array}{c}
6 a_{7}-6 a_{2} a_{6}+6 a_{2}^{2} a_{5}-6 a_{3} a_{5}+12 a_{2} a_{3} a_{4}-a_{2}^{6}-6 a_{2}^{3} a_{4} \\
-3 a_{4}^{2}+2 a_{3}^{3}-9 a_{2}^{2} a_{3}^{2}+6 a_{2}^{4} a_{3}
\end{array}\right) z^{6} \\
& +\cdots
\end{aligned}
$$

On equating coefficients of (35) and (36), we get

$$
\begin{gathered}
a_{2}=\frac{1}{4} c_{1}, \\
a_{3}=\frac{1}{8} c_{2}-\frac{1}{32} c_{1}^{2}, \\
a_{4}=\frac{7}{1152} c_{1}^{3}-\frac{5}{96} c_{2} c_{1}+\frac{1}{12} c_{3}, \\
a_{5}=-\frac{17}{18432} c_{1}^{4}+\frac{7}{384} c_{1}^{2} c_{2}-\frac{1}{24} c_{3} c_{1}-\frac{3}{128} c_{2}^{2}+\frac{1}{16} c_{4},
\end{gathered}
$$

$$
\begin{aligned}
a_{6}= & -\frac{257}{1843200} c_{1}^{5}-\frac{107}{23040} c_{1}^{3} c_{2}+\frac{31}{1920} c_{3} c_{1}^{2}+\frac{139}{7680} c_{1} c_{2}^{2} \\
& -\frac{11}{320} c_{4} c_{1}-\frac{19}{480} c_{3} c_{2}+\frac{1}{20} c_{5},
\end{aligned}
$$

$$
\begin{aligned}
a_{7}= & \frac{33599}{132710400} c_{1}^{6}-\frac{73}{2211840} c_{1}^{4} c_{2}-\frac{79}{17280} c_{1}^{3} c_{3} \\
& -\frac{1451}{184320} c_{1}^{2} c_{2}^{2}+\frac{109}{7680} c_{4} c_{1}^{2}+\frac{47}{1440} c_{1} c_{2} c_{3}-\frac{7}{240} c_{5} c_{1} \\
& +\frac{55}{9216} c_{2}^{3}-\frac{13}{384} c_{4} c_{2}-\frac{5}{288} c_{3}^{2}+\frac{1}{24} c_{6} .
\end{aligned}
$$

Now from (41), we have

$$
\begin{aligned}
& \left|a_{6}\right|=\left|\begin{array}{c}
\frac{1}{96}\left(c_{5}-\frac{9}{10} c_{1} c_{4}\right)+\frac{19}{480}\left(c_{5}-c_{1} c_{4}\right)+\frac{31}{1920} c_{1}^{2}\left(c_{3}-\frac{107}{372} c_{1} c_{2}\right) \\
-\frac{257}{1843200} c_{1}^{5}+\frac{139}{7680} c_{1} c_{2}
\end{array}\right| \\
& \leq \frac{1}{96}\left|c_{5}-\frac{9}{10} c_{1} c_{4}\right|+\frac{19}{480}\left|c_{5}-c_{1} c_{4}\right|+\frac{31}{1920}\left|c_{1}\right|^{2}\left|c_{3}-\frac{107}{372} c_{1} c_{2}\right| \\
& +\frac{257}{1843200}\left|c_{1}\right|^{5}+\frac{139}{7680}\left|c_{1}\right|\left|c_{2}\right| \text {. }
\end{aligned}
$$

By applying (20) and (21) to above we get

$$
\left|a_{6}\right| \leq \frac{21797}{57600}
$$

Now from (42), we have

$$
\begin{aligned}
& \left|a_{7}\right|=\left|\begin{array}{c}
\frac{1}{24}\left(c_{6}-\frac{5}{12} c_{3}^{2}\right)-\frac{13}{384} c_{2}\left(c_{4}-\frac{55}{312} c_{2}^{2}\right)-\frac{7}{240} c_{1}\left(c_{5}-\frac{109}{224} c_{1} c_{4}\right) \\
+\frac{47}{1440} c_{1} c_{2}\left(c_{3}-\frac{1451}{6016} c_{1} c_{2}\right)+\frac{33599}{132710400} c_{1}^{6}-\frac{73}{2211840} c_{1}^{4} c_{2}-\frac{79}{17280} c_{1}^{3} c_{3}
\end{array}\right| \\
& \leq \frac{1}{24}\left|c_{6}-\frac{5}{12} c_{3}^{2}\right|+\frac{13}{384}\left|c_{2}\right|\left|c_{4}-\frac{55}{312} c_{2}^{2}\right|+\frac{7}{240}\left|c_{1}\right|\left|c_{5}-\frac{109}{224} c_{1} c_{4}\right|+\frac{47}{1440}\left|c_{1}\right|\left|c_{2}\right| \\
& \cdot\left|c_{3}-\frac{1451}{6016} c_{1} c_{2}\right|+\frac{33599}{132710400}\left|c_{1}\right|^{6}+\frac{73}{2211840}\left|c_{1}\right|^{4}\left|c_{2}\right|+\frac{79}{17280}\left|c_{1}\right|^{3}\left|c_{3}\right| \text {. }
\end{aligned}
$$

By applying (20) and (21) to the above, we get

$$
\left|a_{7}\right| \leq \frac{1424429}{2073600}
$$

Now, from (37)-(39), we have

$$
\left|a_{2} a_{4}-a_{3}^{2}\right|=\left|\frac{5}{9216} c_{1}^{4}-\frac{1}{192} c_{1}^{2} c_{2}+\frac{1}{48} c_{3} c_{1}-\frac{1}{64} c_{2}^{2}\right| .
$$


Using Lemma 2, we get

$$
\begin{aligned}
\left|a_{2} a_{4}-a_{3}^{2}\right|= & \mid-\frac{7}{9216} c_{1}^{4}-\frac{1}{192} c_{1}^{2} x^{2}\left(4-c_{1}^{2}\right)-\frac{1}{256} x^{2}\left(4-c_{1}^{2}\right)^{2} \\
& +\frac{1}{96} c_{1}\left(4-c_{1}^{2}\right)\left(1-|x|^{2}\right) z \mid
\end{aligned}
$$

Let $|x|=y, y \in[0,1], c_{1}=c, c \in[0,2]$, and $|z|=1$, along with triangle inequality, and we have

$$
\begin{aligned}
\left|a_{2} a_{4}-a_{3}^{2}\right| \leq & \frac{7}{9216} c^{4}+\frac{1}{192} c^{2} y^{2}\left(4-c^{2}\right)+\frac{1}{256} y^{2}\left(4-c^{2}\right)^{2} \\
& +\frac{1}{96} c\left(4-c^{2}\right)\left(1-y^{2}\right)=G(c, y) .
\end{aligned}
$$

Differentiating (49) partially with respect to $y$, we have

$$
\frac{\partial G(c, y)}{\partial y}=\frac{\left(c^{2}-8 c+12\right)\left(4-c^{2}\right) y}{384}>0
$$

showing that $G(c, y)$ is an increasing function in interval $y \in[0,1], c \in[0,2]$,so the maximum is attained at $y=1$, that is,

$$
\begin{aligned}
\max G(c, y)= & G(c, 1)=\frac{7}{9216} c^{4}+\frac{1}{192} c^{2}\left(4-c^{2}\right) \\
& +\frac{1}{256}\left(4-c^{2}\right)^{2}=F(c)
\end{aligned}
$$

Now

$$
\begin{aligned}
F^{\prime}(c) & =\frac{7}{2304} c^{3}+\frac{1}{96} c\left(4-c^{2}\right)-\frac{1}{96} c^{3}-\frac{1}{64} c\left(4-c^{2}\right) \\
& =\frac{7}{2304} c^{3}-\frac{1}{192} c\left(4-c^{2}\right)-\frac{1}{96} c^{3},
\end{aligned}
$$

since $F^{\prime}(c)=0$ has root at $c=0$ and also

$$
F^{\prime \prime}(c)=-\frac{1}{48}<0
$$

so the maximum is attained at $c=0$; therefore, we have

$$
F(c)=\frac{1}{16}
$$

Hence,

$$
\left|a_{2} a_{4}-a_{3}^{2}\right| \leq F(c)=\frac{1}{16}
$$

For the third Hankel determinant, we need the following result.
Lemma 7 (see [17]). Let $f \in \mathcal{S}_{S G}^{*}$ be of the form (2). Then,

$$
\begin{aligned}
& \left|a_{2}\right| \leq \frac{1}{2}, \\
& \left|a_{3}\right| \leq \frac{1}{4}, \\
& \left|a_{4}\right| \leq \frac{1}{6} \\
& \left|a_{5}\right| \leq \frac{1}{8} .
\end{aligned}
$$

Theorem 8. Let $f \in \mathcal{S}_{S G}^{*}$ be of the form (2). Then,

$$
\left|H_{3,1}(f)\right| \leq \frac{43}{576} \simeq 0.07465
$$

Proof. Since from (16), we have

$$
H_{3,1}(f)=a_{3}\left(a_{2} a_{4}-a_{3}^{2}\right)-a_{4}\left(a_{4}-a_{2} a_{3}\right)+a_{5}\left(a_{3}-a_{2}^{2}\right),
$$

by applying triangle inequality, we obtain

$$
\left|H_{3,1}(f)\right| \leq\left|a_{3}\right|\left|a_{2} a_{4}-a_{3}^{2}\right|+\left|a_{4}\right|\left|a_{4}-a_{2} a_{3}\right|+\left|a_{5}\right|\left|a_{3}-a_{2}^{2}\right| .
$$

Now, using Lemmas 4-7 and Theorem 6 in conjunction with (59), we can get the required result.

\section{Bounds of $H_{4,1}(f)$ for the Class $\mathcal{S}_{S G}^{*}$}

In recent years, researchers has started to evaluate the fourth-order Hankel determinant for different subclasses of analytic functions. The trend of finding the fourth-order Hankel determinant in geometric function theory started in 2018, when Arif et al. [44] studied and obtained the upper bound for the class of bounded turning functions. Recently Zhang and Tang [31] studied the fourth-order Hankel determinant for a subclass of starlike functions associated with the sine function. Inspired from the recent research going on and from the above works, we discuss here the fourthorder Hankel determinant for the class $\mathcal{S}_{S G}^{*}$.

From (15), we can write $H_{4,1}(f)$ as

$$
H_{4,1}(f)=a_{7} H_{3,1}(f)-a_{6} \delta_{1}+a_{5} \delta_{2}-a_{4} \delta_{3}
$$

where

$$
\begin{aligned}
& \delta_{1}=a_{3}\left(a_{2} a_{5}-a_{3} a_{4}\right)-a_{4}\left(a_{5}-a_{2} a_{4}\right)+a_{6}\left(a_{3}-a_{2}^{2}\right), \\
& \delta_{2}=a_{3}\left(a_{3} a_{5}-a_{4}^{2}\right)-a_{5}\left(a_{5}-a_{2} a_{4}\right)+a_{6}\left(a_{4}-a_{2} a_{3}\right), \\
& \delta_{3}=a_{4}\left(a_{3} a_{5}-a_{4}^{2}\right)-a_{5}\left(a_{2} a_{5}-a_{3} a_{4}\right)+a_{6}\left(a_{4}-a_{2} a_{3}\right) .
\end{aligned}
$$


Theorem 9. Let $f \in \mathcal{S}_{S G}^{*}$ be of the form (2), and then,

$$
\left|a_{2} a_{5}-a_{3} a_{4}\right| \leq \frac{5}{36}
$$

Proof. From equations (37), (38), (39), and (40), we get

$$
\begin{aligned}
\left|a_{2} a_{5}-a_{3} a_{4}\right|=\mid & -\frac{1}{24576} c_{1}^{5}+\frac{5}{2304} c_{1}^{3} c_{2}-\frac{1}{128} c_{3} c_{1}^{2}+\frac{1}{1536} c_{1} c_{2}^{2} \\
& +\frac{1}{64} c_{4} c_{1}-\frac{1}{96} c_{3} c_{2}|=| \frac{5}{2304} c_{1}^{3}\left(c_{2}-\frac{3}{360} c_{1}^{2}\right) \\
& +\frac{1}{64} c_{1}\left(c_{4}-\frac{1}{2} c_{1} c_{3}\right)-\frac{1}{96} c_{2}\left(c_{3}-\frac{1}{16} c_{1} c_{2}\right) \mid \\
\leq & \frac{5}{2304}\left|c_{1}\right|^{3}\left|c_{2}-\frac{3}{360} c_{1}^{2}\right|+\frac{1}{64}\left|c_{1}\right|\left|c_{4}-\frac{1}{2} c_{1} c_{3}\right| \\
& +\frac{1}{96}\left|c_{2}\right|\left|c_{3}-\frac{1}{16} c_{1} c_{2}\right|
\end{aligned}
$$

Now, making use of (20), (21), and (22) in conjunction with (65), we can get the required result.

Theorem 10. Let $f \in \mathcal{S}_{S G}^{*}$ be of the form (2), and then,

$$
\left|a_{5}-a_{2} a_{4}\right| \leq \frac{1}{8}
$$

Proof. From equations (37), (39), and (40), we get

$$
\left|a_{5}-a_{2} a_{4}\right|=\frac{1}{16}\left|\frac{5}{128} c_{1}^{4}-\frac{1}{2} c_{1}^{2} c_{2}+c_{3} c_{1}+\frac{3}{8} c_{2}^{2}-c_{4}\right| .
$$
result.

Applying Lemma 3 to the last term, we get the required

Theorem 11. Let $f \in \mathcal{S}_{S G}^{*}$ be of the form (2), and then,

$$
\left|a_{3} a_{5}-a_{4}^{2}\right| \leq \frac{7405}{82944}
$$

Proof. From equations (37), (39), and (40), we get

$$
\begin{aligned}
\left|a_{3} a_{5}-a_{4}^{2}\right|=\mid & -\frac{43}{5308416} c_{1}^{6}-\frac{23}{442368} c_{1}^{4} c_{2}+\frac{1}{3456} c_{1}^{3} c_{3} \\
& +\frac{11}{36864} c_{1}^{2} c_{2}^{2}-\frac{1}{512} c_{4} c_{1}^{2}+\frac{1}{288} c_{1} c_{2} c_{3}-\frac{3}{1024} c_{2}^{3} \\
& +\frac{1}{128} c_{4} c_{2}-\frac{1}{144} c_{3}^{2}|=|-\frac{43}{5308416} c_{1}^{6} \\
& -\frac{23}{442368} c_{1}^{4} c_{2}-\frac{1}{144} c_{3}\left(c_{3}-\frac{1}{2} c_{1} c_{2}\right) \\
& -\frac{3}{1024} c_{2}^{2}\left(c_{2}-\frac{11}{108} c_{1}^{2}\right)+\frac{1}{128} c_{4}\left(c_{2}-\frac{1}{4} c_{1}^{2}\right)
\end{aligned}
$$

$$
\begin{aligned}
& +\left.\frac{1}{3456} c_{1}^{3}\left(c_{3}-\frac{23}{128} c_{1} c_{2}\right)\left|\leq \frac{43}{5308416}\right| c_{1}\right|^{6} \\
& +\frac{23}{442368}\left|c_{1}\right|^{4}\left|c_{2}\right|+\frac{1}{144}\left|c_{3}\right|\left|c_{3}-\frac{1}{2} c_{1} c_{2}\right| \\
& +\frac{3}{1024}\left|c_{2}\right|^{2}\left|c_{2}-\frac{11}{108} c_{1}^{2}\right|+\frac{1}{128}\left|c_{4}\right|\left|c_{2}-\frac{1}{4} c_{1}^{2}\right| \\
& +\frac{1}{3456}\left|c_{1}\right|^{3}\left|c_{3}-\frac{23}{128} c_{1} c_{2}\right| .
\end{aligned}
$$

Now, making use of (20), (21), and (22) in conjunction with (69), we can get the required result.

Theorem 12. Let $f \in \mathcal{S}_{S G}^{*}$ be of the form (2), and then,

$$
\left|H_{4,1}(f)\right| \leq \frac{16431024581}{119439360000} \simeq 0.13757
$$

Proof. From (15), we have

$$
H_{4,1}(f)=a_{7} H_{3,1}(f)-a_{6} \delta_{1}+a_{5} \delta_{2}-a_{4} \delta_{3}
$$

where $\delta_{1}, \delta_{2}$, and $\delta_{3}$ are defined in (61), (62), and (63), respectively. Now, using triangle inequalities, we have

$$
\left|H_{4,1}(f)\right| \leq\left|a_{7}\right|\left|H_{3,1}(f)\right|+\left|a_{6}\right|\left|\delta_{1}\right|+\left|a_{5}\right|\left|\delta_{2}\right|+\left|a_{4}\right|\left|\delta_{3}\right|,
$$

since

$$
\begin{aligned}
\left|\delta_{1}\right| & =\left|a_{3}\left(a_{2} a_{5}-a_{3} a_{4}\right)-a_{4}\left(a_{5}-a_{2} a_{4}\right)+a_{6}\left(a_{3}-a_{2}^{2}\right)\right| \\
& \leq\left|a_{3}\right|\left|a_{2} a_{5}-a_{3} a_{4}\right|+\left|a_{4}\right|\left|a_{5}-a_{2} a_{4}\right|+\left|a_{6}\right|\left|a_{3}-a_{2}^{2}\right| .
\end{aligned}
$$

By applying Lemmas 4 and 7 and Theorems 6, 9, and 10, we get

$$
\left|\delta_{1}\right| \leq \frac{34597}{230400}
$$

And also,

$$
\begin{aligned}
\left|\delta_{2}\right| & =\left|a_{3}\left(a_{3} a_{5}-a_{4}^{2}\right)-a_{5}\left(a_{5}-a_{2} a_{4}\right)+a_{6}\left(a_{4}-a_{2} a_{3}\right)\right| \\
& \leq\left|a_{3}\right|\left|a_{3} a_{5}-a_{4}^{2}\right|+\left|a_{5}\right|\left|a_{5}-a_{2} a_{4}\right|+\left|a_{6}\right|\left|a_{4}-a_{2} a_{3}\right| .
\end{aligned}
$$

Using Lemmas 5 and 7 and Theorems 6, 10, and 11 we get

$$
\left|\delta_{2}\right| \leq \frac{837853}{8294400}
$$


Also, again,

$$
\begin{aligned}
\left|\delta_{3}\right| & =\left|a_{4}\left(a_{3} a_{5}-a_{4}^{2}\right)-a_{5}\left(a_{2} a_{5}-a_{3} a_{4}\right)+a_{6}\left(a_{4}-a_{2} a_{3}\right)\right| \\
& \leq\left|a_{4}\right|\left|a_{3} a_{5}-a_{4}^{2}\right|+\left|a_{5}\right|\left|a_{2} a_{5}-a_{3} a_{4}\right|+\left|a_{6}\right|\left|a_{4}-a_{2} a_{3}\right|,
\end{aligned}
$$
get

Using Lemmas 5 and 7 and Theorems 6, 9, and 11, we

$$
\left|\delta_{3}\right| \leq \frac{1185817}{12441600} .
$$

Now, using the values of (74), (76), and (78) along with Theorem 6 and Lemma 7 to (72), we get the desired the estimate.

\section{Zalcman Conjecture for Class $\mathcal{S}_{S G}^{*}$}

One of the main conjectures in the geometric function theory, suggested by Lawrence Zalcman in 1960, is that the coefficients of class $\delta$ satisfy the inequality

$$
\left|a_{n}^{2}-a_{2 n-1}\right| \leq(n-1)^{2} .
$$

Only the well-known Koebe function $k(z)=z /(1-z)^{2}$ and its rotations have equality in the above form. For the popular Fekete-Szego inequality, when $n=2$, the equality holds. Recently, Khan et al. [43] evaluated the Zalcman conjecture for the class of starlike functions with respect to symmetric points associated with the sine function. Many researchers have studied the Zalcman function in the literature [45-47].

Theorem 13. Let $f \in \mathcal{S}_{S G}^{*}$ be of the form (2), and then,

$$
\left|a_{5}-a_{3}^{2}\right| \leq \frac{1}{8}
$$

The result is sharp for function

$$
f(z)=z \exp \left(\int_{0}^{z} \frac{e^{t^{4}}-1}{t\left(e^{t^{4}}+1\right)} d t\right)=z+\frac{1}{8} z^{5}+\cdots
$$

Proof. From equations (37) and (40), we get

$$
\left|a_{5}-a_{3}^{2}\right|=\frac{1}{16}\left|-\frac{35}{1152} c_{1}^{4}+\frac{5}{12} c_{1}^{2} c_{2}-\frac{2}{3} c_{3} c_{1}-\frac{5}{8} c_{2}^{2}+c_{4}\right| .
$$

Using Lemma 3 and equation (82), we can get the required result.

\section{Bounds of $H_{4,1}(f)$ for 2-Fold, 3-Fold, and 4- Fold Symmetric Functions}

Let $m \in \mathbb{N}=\{1,2,3, \cdots$,$\} . It is called m$-fold symmetric if a rotation of domain $\mathbb{U}$ about the origin through an angle $2 \pi$
I $m$ carries itself on the domain $\mathbb{U}$. It is obvious that in $\mathbb{U}$, an analytic function $f$ is $m$-fold symmetric if

$$
f\left(e^{\frac{2 \pi}{m}} z\right)=e^{\frac{2 \pi}{m}} f(z)(\forall z \in \mathbb{U}) .
$$

The set of $m$-fold symmetric univalent functions with the following series:

$$
f(z)=z+\sum_{k=2}^{\infty} a_{m k+1} z^{m k+1}(\forall z \in \mathbb{U})
$$

is referred to as $\delta^{(m)}$.

The subclass $\mathcal{S}_{S G}^{*(m)}$ is a collection of $m$-fold symmetric starlike functions associated with the modified sigmoid function. More precisely, an analytic function $f$ of the form (84) belongs to class $\mathcal{S}_{S G}^{*(m)}$ if and only if

$$
\frac{z f^{\prime}(z)}{f(z)}=\frac{2}{1+e^{-((p(z)-1) / p(z)+1)}}\left(p \in \mathscr{P}^{(m)}\right),
$$

where the set $\mathscr{P}^{(m)}$ is defined by

$$
\mathscr{P}^{(m)}=\left\{p \in \mathscr{P}: p(z)=1+\sum_{k=1}^{\infty} c_{m k} z^{m k}(\forall z \in \mathbb{U})\right\} .
$$

Theorem 14. If $f \in \mathcal{S}_{S G}^{*(2)}$ and be of the form (84), then,

$$
\left|a_{3} a_{7}-a_{5}^{2}\right| \leq \frac{751}{21504}
$$

Proof. Since $f \in \mathcal{S}_{S G}^{*(2)}$, therefore, there exists a function $p \in$ $\mathscr{P}^{(2)}$ such that

$$
\frac{z f^{\prime}(z)}{f(z)}=\frac{2}{1+e^{-((p(z)-1) / p(z)+1)}} .
$$

Using the series forms (84) and (86), when $m=2$ in the above relation, we have

$$
a_{3}=\frac{1}{8} c_{2}
$$

$$
a_{5}=\frac{1}{16} c_{4}-\frac{3}{128} c_{2}^{2}
$$

$$
a_{7}=\frac{55}{10752} c_{2}^{3}-\frac{13}{448} c_{4} c_{2}+\frac{1}{28} c_{6} .
$$


Now, using (89), (90), and (91), we get

$$
\begin{aligned}
\left|a_{3} a_{7}-a_{5}^{2}\right| & =\left|\frac{31}{344064} c_{2}^{4}-\frac{5}{7168} c_{2}^{2} c_{4}+\frac{1}{224} c_{6} c_{2}-\frac{1}{256} c_{4}^{2}\right| \\
& =\left|\frac{31}{344064} c_{2}^{4}+\frac{1}{224} c_{2}\left(c_{6}-\frac{5}{32} c_{2} c_{4}\right)-\frac{1}{256} c_{4}^{2}\right| \\
& \leq \frac{31}{344064}\left|c_{2}\right|^{4}+\frac{1}{224}\left|c_{2}\right|\left|c_{6}-\frac{5}{32} c_{2} c_{4}\right|+\frac{1}{256}\left|c_{4}\right|^{2} .
\end{aligned}
$$

Now, using (20) and (21) to the above, we get the required result.

Theorem 15. If $f \in \mathcal{S}_{S G}^{*(2)}$ and be of the form (84), then,

$$
\left|a_{5}-a_{3}^{2}\right| \leq \frac{1}{8}
$$

Proof. Using (89) and (90), we have

$$
\left|a_{5}-a_{3}^{2}\right|=\frac{1}{16}\left|c_{4}-\frac{5}{8} c_{2}^{2}\right|
$$

Using (21) to the above, we get the required result.

Theorem 16. If $f \in \mathcal{S}_{S G}^{*(2)}$ and be of the form (84), then,

$$
\left|H_{4,1}(f)\right| \leq \frac{751}{172032}
$$

Proof. Since $f \in \mathcal{S}_{S G}^{*(2)}$, therefore, $a_{2}=a_{4}=a_{4}=0$ and we have

$$
H_{4,1}(f)=\left(a_{5}-a_{3}^{2}\right)\left(a_{3} a_{7}-a_{5}^{2}\right) .
$$

Then,

$$
\left|H_{3,1}(f)\right|=\left|a_{5}-a_{3}^{2}\right|\left|a_{3} a_{7}-a_{5}^{2}\right|,
$$

Using (87) and (93), we get the required result.

Theorem 17. If $f \in \mathcal{S}_{S G}^{*(3)}$ and be of the form (84), then,

$$
\left|H_{4,1}(f)\right| \leq \frac{1}{432}
$$

Proof. Since $f \in \mathcal{S}_{S G}^{*(3)}$, therefore, there exists a function $p \in$ $\mathscr{P}^{(3)}$ such that

$$
\frac{z f^{\prime}(z)}{f(z)}=\frac{2}{1+e^{-((p(z)-1) / p(z)+1)}} .
$$

Using the series forms (84) and (86), when $m=3$ in the above relation, we have

$$
\begin{gathered}
a_{4}=\frac{1}{12} c_{3}, \\
a_{7}=\frac{1}{24} c_{6}-\frac{5}{288} c_{3}^{2} .
\end{gathered}
$$

Now,

$$
H_{4,1}(f)=a_{4}^{2}\left(a_{4}^{2}-a_{7}\right)
$$

Therefore,

$\left|H_{4,1}(f)\right|=\left|-\frac{1}{3456} c_{3}^{2}\left(c_{6}-\frac{7}{12} c_{3}^{2}\right)\right|=\frac{1}{3456}\left|c_{3}\right|^{2}\left|c_{6}-\frac{7}{12} c_{3}^{2}\right|$.

Using (20) and (21), we get the desired result.

Theorem 18. If $f \in \mathcal{S}_{S G}^{*(4)}$ and be of the form (84), then,

$$
\left|H_{4,1}(f)\right| \leq \frac{1}{64}
$$

Proof. Since $f \in \mathcal{S}_{S G}^{*(4)}$, therefore, there exists a function $p \in$ $\mathscr{P}^{(4)}$ such that

$$
\frac{z f^{\prime}(z)}{f(z)}=\frac{2}{1+e^{-((p(z)-1) / p(z)+1)}}
$$

Using the series forms (84) and (86), when $m=4$ in the above relation, we have

$$
a_{5}=\frac{1}{16} c_{4}
$$

Since $f \in \mathcal{S}_{S G}^{*(4)}$, therefore, $a_{2}=a_{3}=a_{4}=a_{6}=a_{7}=0$ and we have

$$
\begin{gathered}
H_{4,1}(f)=a_{5}^{2}, \\
H_{4,1}(f)=\frac{1}{256} c_{4}^{2} .
\end{gathered}
$$

Now,

$$
\left|H_{4,1}(f)\right|=\frac{1}{256}\left|c_{4}\right|^{2}
$$

Using (20) to the above, we get the required result.

\section{Conclusion}

In our present investigation, we have obtained the improved third-order Hankel determinant for a class of starlike functions connected with modified sigmoid functions. Furthermore, we have investigated the fourth-order Hankel determinant and Zalcman conjecture and also evaluated fourth-order Hankel determinants for 2-fold, 3-fold, and 4fold symmetric starlike functions. 


\section{Data Availability}

No data were used to support this study.

\section{Conflicts of Interest}

The authors declare that they have no conflicts of interest.

\section{Authors' Contributions}

All authors equally contributed to this manuscript and approved the final version.

\section{References}

[1] L. De Branges, "A proof of the Bieberbach conjecture," Acta Mathematica, vol. 154, no. 1-2, pp. 137-152, 1985.

[2] S. S. Miller and P. T. Mocanu, "Second order differential inequalities in the complex plane," Journal of Mathematical Analysis and Applications, vol. 65, no. 2, pp. 289-305, 1978.

[3] S. S. Miller, P. T. Mocanu, and Differential Subordinations, Theory and Applications, Series on Monographs and Textbooks in Pure and Appl. Math. No. 225, Marcel Dekker Inc., New York, NY, USA, 2000.

[4] W. Janowski, "Extremal problems for a family of functions with positive real part and for some related families," Annales Polonici Mathematici, vol. 23, no. 2, pp. 159-177, 1970.

[5] M. S. Robertson, "Certain classes of starlike functions," Michigan Mathematical Journal, vol. 32, no. 2, pp. 135-140, 1985.

[6] F. Ronning, "Uniformly convex functions and a corresponding class of starlike functions," Proceedings of American Mathematical Society, vol. 118, no. 1, pp. 189-196, 1993.

[7] A. Jangteng, S. A. Halim, and M. Darus, "Coefficient inequality for starlike and convex functions," International Journal of Mathematical Analysis, vol. 1, pp. 619-625, 2007.

[8] J. Sokół, "Radius problems in the class SL," Applied Mathematics and Computation, vol. 214, no. 2, pp. 569-573, 2009.

[9] N. E. Cho, V. Kumar, S. Kumar, and V. Ravichandran, "Radius problems for starlike functions associated with the sine function," Iranian Mathematical Society, vol. 45, no. 1, pp. 213232, 2019.

[10] R. Mendiratta, S. Nagpal, and V. Ravichandran, "On a subclass of strongly starlike functions associated with exponential function," Bulletin of the Australian Mathematical Society, vol. 38, no. 1, pp. 365-386, 2015.

[11] K. Sharma, N. K. Jain, and V. Ravichandran, "Starlike functions associated with a cardioid," Afrika Matematika, vol. 27, no. 5-6, pp. 923-939, 2016.

[12] N. E. Cho, S. Kumar, V. Kumar, V. Ravichandran, and H. M. Srivastava, "Starlike functions related to the Bell numbers," Symmetry, vol. 11, no. 2, 2019.

[13] J. Dzoik, R. K. Raina, and J. Sokół, "On a class of starlike functions related to a shell-like curve connected with Fibonacci numbers," Mathematical and Computer Modelling, vol. 57, no. 5-6, pp. 1203-1211, 2013.

[14] S. Kanas and D. Răducanu, "Some class of analytic functions related to conic domains," Mathematica Slovaca, vol. 64, no. 5, pp. 1183-1196, 2014.

[15] S. Kumar and V. Ravichandran, "A subclass starlike functions associated with rational function," Southeast Asian Bulletin of Mathematics, vol. 40, pp. 199-212, 2016.
[16] W. Ma and M. Minda, "A unified treatment of some special classes of univalent functions," Proceedings of the Conference on Complex Analysis, Z. Li, F. Ren, L. Yang, and S. Zhang, Eds., , pp. 157-169, International Press, Cambridge, MA, USA, 1992.

[17] P. Goel and S. Kumar, "Certain class of starlike functions associated with modified sigmoid function," Bulletin of the Malaysian Mathematical Sciences Society, vol. 43, pp. 957-991, 2020.

[18] C. Pommerenke, "On the coefficients and Hankel determinants of univalent functions," Journal of the London Mathematical Society, vol. s1-41, no. 1, pp. 111-122, 1966.

[19] C. Pommerenke, "On the Hankel determinants of univalent functions," Mathematika, vol. 14, no. 1, pp. 108-112, 1967.

[20] A. Jangteng, S. A. Halim, and M. Darus, "Coefficient inequality for a function whose derivative has a positive real part," Journal of Inequalities in Pure and Applied Mathematics, vol. 7, pp. 1-5, 2006.

[21] D. Răducanu and P. Zaprawa, "Deuxieme determinant de Hankel pour les fonctions presque convexes," Comptes Rendus Mathematique, vol. 355, no. 10, pp. 1063-1071, 2017.

[22] D. V. Krishna and T. RamReddy, "Second Hankel determinant for the class of Bazilevic functions," Universitatis Babeș-Bolyai, vol. 60, pp. 413-420, 2015.

[23] D. Bansal, "Upper bound of second Hankel determinant for a new class of analytic functions," Applied Mathematics Letters, vol. 26, no. 1, pp. 103-107, 2013.

[24] S. K. Lee, V. Ravichandran, and S. Supramaniam, "Bounds for the second Hankel determinant of certain univalent functions," Journal of Inequalities and Applications, vol. 2013, no. 1, 2013.

[25] M. S. Liu, J. F. Xu, and M. Yang, "Upper bound of second Hankel determinant for certain subclasses of analytic functions," Abstract and Applied Analysis, vol. 2014, Article ID 603180, 10 pages, 2014

[26] J. W. Noonan and D. K. Thomas, "On the second Hankel determinant of areally mean $p$-valent functions," Transactions of the American Mathematical Society, vol. 223, pp. 337-346, 1976.

[27] H. Orhan, N. Magesh, and J. Yamini, "Bounds for the second Hankel determinant of certain bi-univalent functions," Turkish Journal of Mathematics, vol. 40, pp. 679-687, 2016.

[28] K. O. Babalola, "On $H_{3}$ (1) Hankel determinant for some classes of univalent functions," http://arxiv.org/abs/0910.3779.

[29] M. Arif, K. I. Noor, and M. Raza, "Hankel determinant problem of a subclass of analytic functions," Journal of Inequalities and Applications, vol. 2012, no. 1, 2012.

[30] L. Shi, M. G. Khan, B. Ahmad, W. K. Mashwani, P. Agarwal, and S. Momani, "Certain coefficient estimate problems for three-leaf-type starlike functions," Fractal and Fractional, vol. 5, no. 4, p. 137, 2021.

[31] H. Y. Zhang and H. A. Tang, "A study of fourth-order Hankel determinants for starlike functions connected with the sine function," Journal of Function Spaces, vol. 2021, Article ID 9991460, 8 pages, 2021.

[32] Q. Hu, H. M. Srivastava, B. Ahmad et al., "A subclass of multivalent Janowski type q-starlike functions and its consequences," Symmetry, vol. 13, no. 7, p. 1275, 2021.

[33] M. G. Khan, B. Ahmad, N. Khan et al., "Applications of Mittag-Leffer type Poisson distribution to a subclass of analytic functions involving conic-type regions," Journal of Function Spaces, vol. 2021, Article ID 4343163, 9 pages, 2021. 
[34] M. Raza and S. N. Malik, "Upper bound of the third Hankel determinant for a class of analytic functions related with lemniscate of Bernoulli," Journal of Inequalities and Applications, vol. 2013, no. 1, 2013.

[35] G. Shanmugam, B. A. Stephen, and K. O. Babalola, "Third Hankel determinant for $\alpha$-starlike functions," Gulf Journal of Mathematics, vol. 2, pp. 107-113, 2014.

[36] P. Zaprawa, "Third Hankel determinants for subclasses of univalent functions," Mediterranean Journal of Mathematics, vol. 14 , no. 1, 2017.

[37] B. Kowalczyk, A. Lecko, and Y. J. Sim, "The sharp bound for the Hankel determinant of the third kind for convex functions," Bulletin of the Australian Mathematical Society, vol. 97, no. 3, pp. 435-445, 2018.

[38] A. Lecko, Y. J. Sim, and B. Śmiarowska, "The sharp bound of the Hankel determinant of the third kind for starlike functions of order 1/2," Complex Analysis and Operator Theory, vol. 13, no. 5, pp. 2231-2238, 2019.

[39] F. Keogh and E. Merkes, "A coefficient inequality for certain classes of analytic functions," Proceedings of the American Mathematical Society, vol. 20, no. 1, pp. 8-12, 1969.

[40] C. Pommerenke, Univalent Functions, Math, Lehrbucher, vandenhoeck and Ruprecht, Gottingen, 1975.

[41] R. J. Libera and E. J. Złotkiewicz, "Early coefficients of the inverse of a regular convex function," Proceedings of the American Mathematical Society, vol. 85, no. 2, pp. 225-230, 1982.

[42] V. Ravichandran and S. Verma, "Borne pour le cinquieme coefficient des fonctions etoilees," Comptes Rendus Mathematique, vol. 353, no. 6, pp. 505-510, 2015.

[43] G. K. Khan, B. Ahmad, G. Murugusundaramoorthy, R. Chinram, and W. K. Mashwani, "Applications of modified Sigmoid functions to a class of starlike functions," Journal of Function Spaces, vol. 2020, Article ID 8844814, 8 pages, 2020.

[44] M. Arif, L. Rani, M. Raza, and P. Zaprawa, "Fourth Hankel determinant for the family of functions with bounded turning," Bulletin of the Korean Mathematical Society, vol. 55, pp. 1703-1711, 2018.

[45] D. Bansal and S. Janusz, "Zalcman conjecture for some subclass of analytic functions," Journal of Fractional Calculus and Application, vol. 8, no. 1, pp. 1-5, 2017.

[46] J. E. Brown and A. Tsao, "On the Zalcman conjecture for starlike and typically real functions," Mathematische Zeitschrift, vol. 191, no. 3, pp. 467-474, 1986.

[47] W. C. Ma, "The Zalcman conjecture for close-to-convex functions," Proceedings of the American Mathematical Society, vol. 104, no. 3, pp. 741-744, 1988. 